\title{
On matchings in hypergraphs
}

\author{
Peter Frankl \\ Tokyo, Japan \\ peter.frankl@gmail.com \\ Tomasz Łuczak* \\ Adam Mickiewicz University \\ Faculty of Mathematics and CS \\ Poznań, Poland \\ and \\ Emory University \\ Department of Mathematics and CS \\ Atlanta, USA \\ tomasz@amu.edu.pl \\ Katarzyna Mieczkowska \\ Adam Mickiewicz University \\ Faculty of Mathematics and CS \\ Poznań, Poland \\ kaska@amu.edu.pl
}

Submitted: Mar 30, 2012; Accepted: Jun 3, 2012; Published: Jun 13, 2012

Mathematics Subject Classifications: 05C35, 05C65, 05C70.

\begin{abstract}
We show that if the largest matching in a $k$-uniform hypergraph $G$ on $n$ vertices has precisely $s$ edges, and $n>3 k^{2} s / 2 \log k$, then $H$ has at most $\left(\begin{array}{l}n \\ k\end{array}\right)-\left(\begin{array}{c}n-s \\ k\end{array}\right)$ edges and this upper bound is achieved only for hypergraphs in which the set of edges consists of all $k$-subsets which intersect a given set of $s$ vertices.
\end{abstract}

A $k$-uniform hypergraph $G=(V, E)$ is a set of vertices $V \subseteq \mathbb{N}$ together with a family $E$ of $k$-element subsets of $V$, which are called edges. In this note by $v(G)=|V|$ and $e(G)=|E|$ we denote the number of vertices and edges of $G=(V, E)$, respectively. By a matching we mean any family of disjoint edges of $G$, and we denote by $\mu(G)$ the size of the largest matching contained in $E$. Moreover, by $\nu_{k}(n, s)$ we mean the largest possible number of edges in a $k$-uniform hypergraph $G$ with $v(G)=n$ and $\mu(G)=s$, and by $\mathcal{M}_{k}(n, s)$ we denote the family of the extremal hypergraphs for this problems, i.e. $H \in \mathcal{M}_{k}(n, s)$ if $v(H)=n, \mu(H)=s$, and $e(H)=\nu_{k}(n, s)$. In 1965 Erdös [2] conjectured that, unless $n=2 k$ and $s=1$, all graphs from $\mathcal{M}_{k}(n, s)$ are either cliques, or belong to the family $\operatorname{Cov}_{k}(n, s)$ of hypergraphs on $n$ vertices in which the set of edges consists of

${ }^{*}$ Partially supported by the Foundation for Polish Science and NSF grant DMS-1102086. 
all $k$-subsets which intersect a given subset $S \subseteq V$, with $|S|=s$. This conjecture, which is a natural generalization of Erdös-Gallai result [3] for graphs, has been verified only for $k=3$ (see [5] and [8]). For general $k$ there have been series of results which state that

$$
\mathcal{M}_{k}(n, s)=\operatorname{Cov}_{k}(n, s) \text { for } n \geqslant g(k) s,
$$

where $g(k)$ is some function of $k$. The existence of such $g(k)$ was shown by Erdös [2], then Bollobás, Daykin and Erdős [1] proved that (1) holds whenever $g(k) \geqslant 2 k^{3}$; Frankl and Füredi [6] showed that (1) is true for $g(k) \geqslant 100 k^{2}$ and recently, Huang, Loh, and Sudakov [7] verified (1) for $g(k) \geqslant 3 k^{2}$. The main result of this note slightly improves these bounds and confirms (1) for $g(k) \geqslant 2 k^{2} / \log k$.

Theorem 1. If $k \geqslant 3$ and

$$
n>\frac{2 k^{2} s}{\log k},
$$

then $\mathcal{M}_{k}(n, s)=\operatorname{Cov}_{k}(n, s)$.

In the proof we use the technique of shifting (for details see [4]). Let $G=(V, E)$ be a hypergraph with vertex set $V=\{1,2, \ldots, n\}$, and let $1 \leqslant i<j \leqslant n$. The hypergraph $\mathbf{s h}_{i, j}(G)$ is obtained from $G$ by replacing each edge $e \in E$ such that $j \in e, i \notin e$ and $e_{i j}=e \backslash\{j\} \cup\{i\} \notin E$, by $e_{i j}$. Let $\mathbf{S h}(G)$ denote the hypergraph obtained from $G$ by the maximum sequence of shifts, such that for all possible $i, j$ we have $\mathbf{s h}_{i j}(\mathbf{S h}(G))=\mathbf{S h}(G)$. It is well known and not hard to prove that the following holds (e.g. see [4] or [8]).

Lemma 2. $G \in \mathcal{M}_{k}(n, s)$ if and only if $\boldsymbol{S h}(G) \in \mathcal{M}_{k}(n, s)$.

Lemma 3. Let $G \in \mathcal{M}_{k}(n, s)$ and $n \geqslant 2 k+1$. Then $G \in \operatorname{Cov}_{k}(n, s)$ if and only if $\operatorname{Sh}(G) \in \operatorname{Cov}_{k}(n, s)$.

Thus, it is enough to show Theorem 1 for hypergraphs $G$ for which $\operatorname{Sh}(G)=G$. Let us start with the following observation.

Lemma 4. If $G$ is a hypergraph on vertex set $[n]$ such that $\boldsymbol{S h}(G)=G$ and $\mu(G)=s$, then

$$
G \subseteq \mathcal{A}_{1} \cup \mathcal{A}_{2} \cup \cdots \cup A_{k}
$$

where

$$
\left.\mathcal{A}_{i}=\{A \subseteq[n]:|A|=k, \mid A \cap\{1,2, \ldots, i(s+1)-1)\} \mid \geqslant i\right\}
$$

for $i=1,2, \ldots, k$.

Proof. Note that the set $e_{0}=\{s+1,2 s+2, \ldots, k s+k\}$ is not an edge of $G$. Indeed, in such a case each of the edges $\{i, i+s+1, \ldots, i+(k-1)(s+1)\}, i=1,2, \ldots, s+1$, belongs to $G$ due to the fact that $G=\operatorname{Sh}(G)$ and, clearly, they form a matching of size $s+1$. Now it is enough to observe that all sets which do not dominate $e_{0}$ must belong to $\bigcup_{i=1}^{k} \mathcal{A}_{i}$.

The following numerical consequence of the above result is crucial for our argument. 
Lemma 5. Let $G$ be a hypergraph with vertex set $\{1,2, \ldots, n\}$ such that $\boldsymbol{S h}(G)=G$ and $\mu(G)=s$, where $n \geqslant k(s+1)-1$. Then all except at most $\frac{s(s+1)}{2}\left(\begin{array}{l}n-1 \\ k-2\end{array}\right)$ edges of $G$ intersect $\{1,2, \ldots, s\}$.

Proof. Let $\mathcal{A}=\bigcup_{i=1}^{k} \mathcal{A}_{i}$. Observe first that $|\mathcal{A}|=s\left(\begin{array}{c}n \\ k-1\end{array}\right)$, for $n \geqslant k(s+1)-1$. Indeed, it follows from an easy induction on $k$, and then on $n$. For $k=1$ it is obvious. For $k \geqslant 1$ and $n=k(s+1)-1$ we have clearly $|\mathcal{A}|=\left(\begin{array}{l}n \\ k\end{array}\right)=s\left(\begin{array}{c}n \\ k-1\end{array}\right)$. Now let $k \geqslant 2, n \geqslant k(s+1)$ and split all the sets of $\mathcal{A}$ into those which contain $n$ and those which do not. Then, the inductional hypothesis gives

$$
|\mathcal{A}|=s\left(\begin{array}{l}
n-1 \\
k-2
\end{array}\right)+s\left(\begin{array}{l}
n-1 \\
k-1
\end{array}\right)=s\left(\begin{array}{c}
n \\
k-1
\end{array}\right) .
$$

Observe also that $\left(\begin{array}{l}n \\ k\end{array}\right)=\sum_{i=1}^{s}\left(\begin{array}{c}n-i \\ k-1\end{array}\right)+\left(\begin{array}{c}n-s \\ k\end{array}\right)$, which is a direct consequence of the identity $\left(\begin{array}{l}n \\ k\end{array}\right)=\left(\begin{array}{l}n-1 \\ k-1\end{array}\right)+\left(\begin{array}{c}n-1 \\ k\end{array}\right)$. Thus, using Lemma 4 and the above observation, the number of edges of $G$ which do not intersect $\{1,2, \ldots, s\}$ can be bounded in the following way.

$$
\begin{aligned}
|G|-\left|G \cap \mathcal{A}_{1}\right| & \leqslant|\mathcal{A}|-\left|\mathcal{A}_{1}\right|=s\left(\begin{array}{c}
n \\
k-1
\end{array}\right)-\left[\left(\begin{array}{l}
n \\
k
\end{array}\right)-\left(\begin{array}{c}
n-s \\
k
\end{array}\right)\right] \\
& =s\left[\sum_{i=1}^{s}\left(\begin{array}{l}
n-i \\
k-2
\end{array}\right)+\left(\begin{array}{l}
n-s \\
k-1
\end{array}\right)\right]-\sum_{i=1}^{s}\left(\begin{array}{l}
n-i \\
k-1
\end{array}\right) \\
& =s \sum_{i=1}^{s}\left(\begin{array}{l}
n-i \\
k-2
\end{array}\right)-\sum_{i=1}^{s} \sum_{j=1}^{s-i}\left(\begin{array}{c}
n-i-j \\
k-2
\end{array}\right) \\
& =s \sum_{i=1}^{s}\left(\begin{array}{l}
n-i \\
k-2
\end{array}\right)-\sum_{i=2}^{s}(i-1)\left(\begin{array}{c}
n-i \\
k-2
\end{array}\right) \\
& =\sum_{i=1}^{s}(s-i+1)\left(\begin{array}{l}
n-i \\
k-2
\end{array}\right) \leqslant \sum_{i=1}^{s} i\left(\begin{array}{l}
n-1 \\
k-2
\end{array}\right) \\
& =\frac{s(s+1)}{2}\left(\begin{array}{l}
n-1 \\
k-2
\end{array}\right) .
\end{aligned}
$$

Proof of Theorem 1. Let us assume that (2) holds for $G \in \mathcal{M}_{k}(n, s)$. Then, by Lemma 2, the hypergraph $H=\mathbf{S h}(G)$ belongs to $\mathcal{M}_{k}(n, s)$. We shall show that $H \in \operatorname{Cov}_{k}(n, s)$ which, due to Lemma 3, would imply that $G \in \operatorname{Cov}_{k}(n, s)$. Our argument is based on the following two observations. Here and below by the $\operatorname{degree} \operatorname{deg}(i)$ of a vertex $i$ we mean the number of edges containing $i$, and by $V$ and $E$ we denote the sets of vertices and edges of $H$ respectively.

Claim 6. If $s \geqslant 2$, then $\{1, k s+2, k s+3, \ldots, k s+k\} \in E$. 
Proof. Let us assume that the assertion does not hold. We shall show that then $H$ has fewer edges than the graph $H^{\prime}=\left(V, E^{\prime}\right)$ whose edge set consists of all $k$-subsets intersecting $\{1,2, \ldots, s\}$. Let $E_{i}=\left\{\{i\} \cup e^{\prime}: e^{\prime} \subset\{k s+2, \ldots, n\},\left|e^{\prime}\right|=k-1\right\}, i \in[s]$ and observe that the sets $E_{i}$ are pairwise disjoint and $\left|E_{i}\right|=\left(\begin{array}{c}n-k s-1 \\ k-1\end{array}\right)$ for every $i \in[s]$. Moreover, since $H=\mathbf{S h}(H)$ and $\{1, k s+2, k s+3, \ldots, k s+k\} \notin E, E_{1} \cap E=\emptyset$, and so $E_{i} \cap E=\emptyset$ for every $i \in[s]$. Thus,

$$
\begin{aligned}
\left|E^{\prime} \backslash E\right| & \geqslant s\left(\begin{array}{c}
n-k s-1 \\
k-1
\end{array}\right) \\
& \geqslant \frac{s(n-1)_{k-1}}{(k-1) !}\left(1-\frac{k s}{n-k+1}\right)^{k-1},
\end{aligned}
$$

while from Lemma 5 we get

$$
\begin{aligned}
\left|E \backslash E^{\prime}\right| & \leqslant \frac{s(s+1)}{2}\left(\begin{array}{l}
n-1 \\
k-2
\end{array}\right)=\frac{s(n-1)_{k-1}}{(k-1) !} \frac{(s+1)(k-1)}{2(n-k+1)} \\
& \leqslant \frac{s(n-1)_{k-1}}{(k-1) !} \frac{k s}{n-k+1} .
\end{aligned}
$$

Thus,

$$
e\left(H^{\prime}\right)-e(H) \geqslant \frac{s(n-1)_{k-1}}{(k-1) !}\left(\left(1-\frac{k s}{n-k+1}\right)^{k-1}-\frac{k s}{n-k+1}\right) .
$$

Let $x=k s /(n-k+1)$. It is easy to check that for all $k \geqslant 3$ and $x \in(0,0.7 \log k / k)$ we have

$$
(1-x)^{k-1}>x \text {. }
$$

Thus, $e\left(H^{\prime}\right)-e(H)>0$ provided $k^{2} s<0.7 \log k(n-k+1)$, which holds whenever $n \geqslant 2 s k^{2} / \log k$. Thus, since clearly $\mu\left(H^{\prime}\right)=s$, we arrive at contradiction with the assumption that $H \in \mathcal{M}_{k}(n, s)$.

Claim 7. If $s \geqslant 2$ then $\operatorname{deg}(1)=\left(\begin{array}{l}n-1 \\ k-1\end{array}\right)$. In particular, the hypergraph $H^{-}$, obtained from $H$ by deleting the vertex 1 together with all edges it is contained in, belongs to $\mathcal{M}_{k}(n-1, s-1)$.

Proof. Let us assume that there is a $k$-subset of $V$, which contains 1 and is not an edge in $H$. Then, in particular, $e=\{1, n-k+2, \ldots, n\} \notin E$. Let us consider hypergraph $\bar{H}$ obtained from $H$ by adding $e$ to its edge set. Since $H \in \mathcal{M}_{k}(n, s)$, there is a matching of size $s+1$ in $\bar{H}$ containing $e$. Hence, as $H=\mathbf{S h}(H)$, there exists a matching $M$ in $H$ such that $M \subset\{2, \ldots, k s+1\}$. Note however that, by Claim $6, f=\{1, k s+2, k s+3, \ldots, k s+$ $k\} \in E$. But then $M^{\prime}=M \cup\{f\}$ is a matching of size $s+1$ in $H$, contradicting the fact that $H \in \mathcal{M}_{k}(n, s)$. Hence, we must have $\operatorname{deg}(1)=\left(\begin{array}{l}n-1 \\ k-1\end{array}\right)$. Since $n \geqslant k s$, the second part of the assertion is obvious.

Now Theorem 1 follows easily from Claim 7 and the observation that, since $\frac{s-1}{n-1} \leqslant \frac{s}{n}$, if (2) holds then it holds also when $n$ is replaced by $n-1$ and $s$ is replaced by $s-1$. Thus, we can reduce the problem to the case when $s=1$ and use Erdős-Ko-Rado theorem (note that then $\left.n>2 k^{2} / \log k>2 k+1\right)$. 


\section{References}

[1] B. Bollobás, E. Daykin, and P. Erdős, Sets of independent edges of a hypergraph, Quart. J. Math. Oxford Ser. (2), 27:25-32, 1976.

[2] P. Erdős, A problem on independent r-tuples, Ann. Univ. Sci. Budapest. Eötvös Sect. Math., 8:93-95, 1965.

[3] P. Erdős and T. Gallai, On maximal paths and circuits of graphs, Acta Math. Acad. Sci. Hungar. 10:337-356, 1959.

[4] P. Frankl, The shifting technique in extremal set theory. In Surveys in Combinatorics, volume 123 of Lond. Math. Soc. Lect. Note Ser., pages 81-110. Cambridge, 1987.

[5] P. Frankl, On the maximum number of edges in a hypergraph with given matching number, arXiv:1205.6847.

[6] P. Frankl and Z. Füredi, unpublished.

[7] H. Huang, P. Loh, and B. Sudakov, The size of a hypergraph and its matching number, Combinatorics, Probability \& Computing, 21:442-450, 2012.

[8] T. Łuczak, K. Mieczkowska, On Erdős' extremal problem on matchings in hypergraphs, arXiv: 1202.4196. 